\title{
The Chance-Constrained Programming for the Lot-Sizing Problem with Stochastic Demand on Parallel Machines
}

\author{
S. Chotayakul and V. Punyangarm
}

\begin{abstract}
We consider the capacitated lot sizing problem with multiple items, setup time, stochastic demand and unrelated parallel machines. The problem is formulated as a stochastic mixed-integer linear programming model. The demand will be assumed to be a normal distribution. The stochastic constraints are transformed into equivalent deterministic programming ones by using the chance constrained programming approach and then obtain an optimal solution by deterministic mixed-integer linear programming model. The proposed algorithm is evaluated through a numerical example. Computational experiment demonstrates that the proposed method have good-quality result for the test problem.
\end{abstract}

Index Terms-Capacitated lot-sizing problem, stochastic demand, chance-constrained programming, parallel machines.

\section{INTRODUCTION}

This paper studies a lot-sizing problem that consists of determining how much to produce of each item in each of the periods in the planning horizon in order to satisfy the uncertain demand of each items, without exceeding the capacity of the machines and minimize total production costs. This problem studied deals with the production of multiple items in a single stage capacitated lot-sizing problem in the setting of unrelated parallel machines (CLSPP). The stochastic demand of each item is assumed to be normal distributed. Each item can be produced on any of the machines and several items can be produced on the same machine in the same time period. A setup time is incurred before starting production and the setup is sequence independent.

The purpose of this paper is to present the way to solve this problem in form of the stochastic mixed integer programming (SMIP) formulation. The traditional method to solve a stochastic program is to transform it into an equivalent deterministic program and then to obtain an optimal solution by some deterministic algorithms [1], [2]. In this work, the stochastic constraints are transformed into an equivalent deterministic constraint by using the approach of chance-constrained programming $(C C P)$, which was first proposed by Charnes and Cooper [3] and was applied in many areas for optimization under uncertainty. The main contribution of this paper is to propose the CCP approach to solve the stochastic mixed-integer linear programming of the

Manuscript received October 8, 2015; revised December 12, 2015.

The authors are with the Department of Industrial Engineering Srinakharinwirot University, Ongkharak, 12110, Thailand (e-mail: supatchaya@g.swu.ac.th, punyangarm@gmail.com). capacitated lot sizing problem with multiple items, setup time, stochastic demand and unrelated parallel machines.

The paper is organized as follows. In Section II, we provide a literature review on lot-sizing problems on parallel machines. In Section III present the four steps of research methodology including, 1) the data collection process, 2) developing model, developing CLSSP problem in form of SMIP model, an transforming SMIP model to MIP model 3) the computing algorithms, and 4) the decision-making process. In Section V, the illustrative numerical example is presented. Finally, the last Section is the conclusions.

\section{LITERATURE REVIEW}

Lot-sizing problem is production planning problem of order purchasing quantity or order quantity produced [4]. Large lot sizes lead to high inventory levels and decrease the number of order cycles while small lot sizes lead to low inventory levels and increase the number of order cycles. The lot-sizing problem is defined as the determination of the production plan that minimizes the total inventory costs, which consists of unit price, setup cost, and inventory holding cost, to satisfy demand [5]. The first inventory planning model was Economic Order Quantity (EOQ) which was presented by Harris [6]. The assumptions of the classic economic order quantity model are a single-item, an infinite time horizon $(T=+\infty)$, a continuous-time, steady-state demand process, no capacity constraints, no lead time, and no shortages allowed.

The focus of this work is on the single stage, multi-item stochastic lot-sizing model that demand varies over time, also known as the dynamic lot-sizing model. Since the assumptions in the EOQ are very restrictive, Wagner and Whitin [7] presented a simple algorithm for solving the dynamic version of the lot size model under the assumption of replacing deterministic demand in each period with a known demand and introducing time-varying demand for a single product over $T$ periods. This model is usually called the single-item uncapacitated lot-sizing problem (SIULS). The objective of the dynamic lot-sizing problem is to determine production quantities for a set of items in order to meet a time-varying demand pattern in a production-inventory environment.

For the classical dynamic lot-sizing model, the setup cost and selling price of the item were assumed to be constant over all time periods and only inventory management costs were considered. The assumptions for the standard dynamic lot-sizing problem are known demand that varies over time, discrete time, single product, no shortage, and no capacity constraints. 
However, in real-world environments, customer demands are unknown. The literature of the models for production planning under uncertainty was reviewed and classified by Mula et al. [8]. Their literature review that they compiled consists of 87 citations from 1983 to 2004. Most implementations in the dynamic lot-sizing problem with demand uncertainty use deterministic lot-sizing procedures with some additional newsvendor calculations to determine buffer stocks or reorder points which was originally proposed by Silver [9]. Kall and Wallace [10] proposed two stochastic approaches to solve a general optimization problem under uncertainty: the two-stage programming and the other method is the chance-constrained programming. The formulation of two-stage stochastic programming problems was first proposed by Dantzig [11]. In this approach, decision variables are divided into two sets; namely, first-stage and second-stage variables. The first-stage variables have to be fixed based on the available information but for the second-stage variables it is assumed that the uncertainty is removed completely before they have to be decided. The formulation of chance-constrained programming problems was pioneered by Charnes and Cooper [3] as a means of handling uncertainty by specifying a confidence level at which it is desired that the stochastic constraint holds [12].

The CCP is one of the well-known approaches to find the best solution for optimization problems under uncertainty, where the objective function or some of the constraints ensure that the probability of one or more events occurring is less than a prescribed threshold. The aim of the problems of stochastic programming with probabilistic criteria is to determine deterministic equivalents of the original stochastic problems.

The CCP approach can be useful for stochastic mixed integer programming model. It is used to find optimal solutions such that an acceptable level of reliability is achieved. There are many applications for chance constraint in stochastic dynamic lot-sizing problem. Bookbinder and Tan [13] studied the stochastic single-stage lot-sizing problem with time-varying demand. They introduced a deterministic equivalent model with chance constraints for this problem and proposed a heuristic method to solve it. Tarim and Kingsman [14] studied the multi-period single-item inventory lot-sizing problem with stochastic demand under the static-dynamic uncertainty strategy of Bookbinder and Tan [13]. They formulated the problem as a mixed integer programming model under service level constraints. Unlike the Bookbinder and Tan model this formulation simultaneously determines the number and timing of the replenishments and the information necessary to determine the size of the replenishment orders, from the replenishment levels for the periods when stock reviews will take place. Their formulation included the effect of a unit variable purchase/production cost. The problem was reformulated by the chance-constrained model and solved by the two-stage Bookbinder-Tan heuristic. Their test results found that the Bookbinder-Tan heuristic had a cost performance that was close to the optimal solution. Compared to the optimal solution, which was a MIP model, the solution times were fast.

Rossi et al. [15] studied production/inventory control problems with stochastic demand and a known non-stationary probability distribution under service level constraint. They proposed an exact stochastic constraint programming approach based on global chance-constraints which extends the original stochastic constraint programming model. They analyzed the accuracy of the solutions provided by the chance-constraint that computes optimal inventory levels to meet the required service level and the expected total cost associated with them. The results showed that proposed approach is a good trade-off between quality of the solution and efficiency of the search process. Taleizadeh et al. [16] proposed a multi-product inventory control problem with dynamic demands and service-level constraints. Then, the problem was transformed into the deterministic equivalent model with chance constraints. This model became an integer non-linear programming and was solved by a harmony search approach with different sizes (small, medium, large) of problem. Its performances were compared to a genetic and a particle swarm optimization algorithms. The results showed that the proposed harmony search approach performs better than both the genetic and a particle swarm optimization algorithms in term of the objective function values. A novel algorithm of stochastic chance-constrained linear programming and its application was proposed by Ding and Wang [2]. They studied computation problem of stochastic chance-constrained linear programming and proposed a novel modified, simplex algorithm based on stochastic simulation. Their numerical example showed that several simple approaches had been tried to solve the stochastic chance-constrained linear programming. The feasibility and validity of the proposed algorithm were illustrated via numerical examples. The results of the proposed algorithm showed its practicability. Chotayakul et al. [17] proposed a two-echelon inventory problem with single-item capacitated lot-sizing model with stochastic demand. They formulated the problem as a SMIP. Then the model was transformed into an equivalent deterministic constraint by using CCP approach and then this formulation was solved by shortest path reformulation approach. The results of the proposed methods showed its effectiveness.

\section{Research Methodology}

This section proposes the research methodology of this paper. It consists of four parts; (A) the data collection process (B) a developing model (C) computing algorithms and (D) a decision - making process. The steps of each part are as follows.

\section{A. Data Collection Process}

The data collection process is the process to obtain the information which can be used as input parameters in the stochastic lot-sizing model for production planning. The input parameters are as follows:

\section{Parameters}

$d_{i t}$ : stochastic demand of item $i$ in period $t$;

$s_{i j}$ : setup cost of item $i$ on machine $j$;

$p_{i j}$ : unit production cost of item $i$ on machine $j$;

$h_{i}$ : unit inventory cost of item $i$; 
$u_{i}$ : unit cost of initial inventory of item $i$;

$f_{i j}$ : setup time of item $i$ on machine $j$;

$b_{i j}$ : time to produce one unit of item $i$ on machine $j$;

$C_{j t}$ : capacity (in unit of time) of machine $j$ in period $t$;

$M$ : an upper bound on $x_{i j t}$

\section{B. Developing Model}

\section{1) Developing CLSSP problem in form of SMIP model}

The dynamic lot-sizing problem was first proposed by Wagner and Whitin [7]. They introduced a dynamic programming approach based on the Wagner-Whitin (W-W) property to find the solution. The objective of the dynamic lot-sizing problem is to determine production quantities for a set of items to meet a time-varying demand pattern in a production-inventory environment. This part, we develop the problem formulation in form of stochastic mixed-integer program (SMIP) for the capacitated multi-items lot-sizing problem with stochastic demand on parallel machines. A mathematical model for minimizing the sum of production, setup and inventory costs is introduced.

The following notations of decision variables are used in the mathematical formulation of the problem

\section{Decision Variables}

$x_{i j t}$ : amount of unit produced of item $i$ on machine $j$ in period $t$;

$y_{i j t}$ : binary variable, indicating the production or not of item $i$ on machine $j$ in period $t$;

$I_{i t}$ : quantity of inventory of item $i$ at the end of period $t$;

$J_{i 0}$ : initial quantity of inventory of item $i$.

The CLSPP can be formulated as the following SMIP model.

Minimize

$$
\sum_{i=1}^{n} u_{i} J_{i 0}+\sum_{t=1}^{T} \sum_{j=1}^{m} \sum_{i=1}^{n}\left(s_{i j} y_{i j t}+p_{i j} x_{i j t}\right)+\sum_{t=1}^{T} \sum_{i=1}^{n} h_{i} I_{i t}
$$

subject to

$$
\begin{gathered}
\sum_{j=1}^{m} x_{i j t}+I_{i, t-1}-I_{i t} \geq d_{i t} \quad i=1, \ldots, n ; t=1, \ldots, T, \\
\sum_{i=1}^{n}\left(b_{i j} x_{i j t}+f_{i j} y_{i j t}\right) \leq C_{j t} \quad j=1, \ldots, m ; t=1, \ldots, T, \\
x_{i j t} \leq M y_{i j t} \quad i=1, \ldots, n ; j=1, \ldots, m ; t=1, \ldots, T, \\
y_{i j t} \in\{0,1\}, x_{i j t} \geq 0, I_{i t} \geq 0 \\
i=1, \ldots, n ; j=1, \ldots, m ; t=1, \ldots, T .
\end{gathered}
$$

The objective function 1) is to minimize the total initial inventory, setup, production, and inventory costs. The constraints 2) guarantee the inventory balance in each period. To avoid infeasible problems, the model considers the possibility of initial inventory that the cost of this inventory is very big. Note that, in general, the inventory balance constraint is initially declared as an equality constraint for deterministic problem, but the inequality constraint is used here due to a stochastic condition. Therefore, in this study, these constraints are changed to " $>$ " by relaxing the upper bound of the constraints in order to maintain feasibility under the stochastic condition and to ensure that supply meets the demand. Constraints 3 ) represent the capacity constraints: the amount of capacity used for production is limited.
Constraints 4) are the machine setup constraints. Finally, constraints 5) define the binary setup variables and non-negative variables for produced quantities and inventory level. These constraints assure that no backlogging occurs.

Since this problem formulation has been shown to be a stochastic mixed-integer program. The basic approach in the stochastic programming is to solve the problem with known deterministic techniques by transforming it from a probabilistic structure to a deterministic structure.

In the next subsection, we will propose steps of transforming SMIP to MIP by using the technique of the chance-constrained programming model, which is the method for dealing with random parameters in optimization problems under uncertainty. This approach can be used to transform a stochastic programming model into an equivalent deterministic model and has been widely used in engineering and financial decision-making problems [18], where an objective function is minimized or maximized under probabilistic constraints. Therefore, the probabilistic constraint, that is the Equation (2) of our problem, will be transformed to the equivalent deterministic constraint. The steps of transforming this chance constraint of our problem will be shown in the next subsection.

\section{2) Transforming SMIP model to MIP model}

In this study, we now force uncertain constraints (In constraints (2), stochastic demands, $d_{i t}$ are normally distributed) as equivalent deterministic constraints by using the CCP approach. The equivalent deterministic model will allow us to use techniques for solving deterministic problems without loss of optimality [19]. At the end of each and every time period, the probability that the items will support customers' need is set to be at lease $1-\alpha_{i t}$. As the chance constraints, the constraints (2) can be written in the following form,

$$
\operatorname{Pr}\left\{d_{i t} \leq \sum_{j=1}^{m} x_{i j t}+I_{i, t-1}-I_{i t}\right\} \geq 1-\alpha_{i t}
$$

Let $E\left(d_{i t}\right)$ be the expected value of $d_{i t}$ and $\operatorname{Var}\left(d_{i t}\right)$ be its variance. The deterministic equivalent of chance constraint (6) is

$$
\operatorname{Pr}\left\{\eta_{i t} \leq \frac{\sum_{j=1}^{m} x_{i j t}+I_{i, t-1}-I_{i t}-E\left(d_{i t}\right)}{\sqrt{\operatorname{Var}\left(d_{i t}\right)}}\right\} \geq 1-\alpha_{i t},
$$

where $\eta_{i t}$ is the standardized normally distributed random variable. The Equation (7) holds if and only if

$$
\frac{\sum_{j=1}^{m} x_{i j t}+I_{i, t-1}-I_{i t}-E\left(d_{i t}\right)}{\sqrt{\operatorname{Var}\left(d_{i t}\right)}} \geq \Phi_{i t}^{-1}\left(1-\alpha_{i t}\right) .
$$

where $\Phi_{i t}^{-1}$ is inverse function of the standardized normal distribution. That is the deterministic equivalent of chance constraints are

$$
\begin{gathered}
\sum_{j=1}^{m} x_{i j t}+I_{i, t-1}-I_{i t} \geq E\left(d_{i t}\right)+\Phi_{i t}^{-1}\left(1-\alpha_{i t}\right) \sqrt{\operatorname{Var}\left(d_{i t}\right)} \\
i=1, \ldots, n ; t=1, \ldots, T .
\end{gathered}
$$

Therefore, the equivalent deterministic programming formulation of the CLSPP problem in form of MIP formulation consists of Equations (1), (3)-(5) and (9). 


\section{Computing Algorithm}

After using the CCP approach to transform the SMIP formulation of CLSPP problem to the MIP formulation, this model can be solved with standard approach and basic software to obtain the optimal solution [20].

\section{Decision-Making Process}

Changing the levels of significance $\left(\alpha_{i}\right)$ affects the probability of stock-out and the inventory. If significance levels increase, the inventory levels will decrease but the probability of stock-out will increase. Inventory planners can make decisions under the acceptable significance levels by trade-off between the probability of stock-out and the inventory level.

The effectiveness of the decision making process in production planning depends on accuracy of input parameters information and the variation of stochastic demand.

\section{An Illustrative NuMERICAL EXAMPLE}

The numerical example of the production planning of multiple items in a single stage capacitated lot-sizing problem in the setting of unrelated parallel machines with stochastic demand is illustrated to show how to use the chance-constrained programming approach to solve this problem.

\section{A. Problem Description}

TABLE I: STOCHASTIC DEMAND FOR ALl ITEMS FOR FOUR PERIODS

\begin{tabular}{llll}
\hline \hline Time & \multicolumn{3}{c}{ Item 1 } \\
\cline { 2 - 4 } Periods & Mean & SD & RHS \\
\hline Week 1 & 200 & 13 & 222 \\
Week 2 & 300 & 17 & 328 \\
Week 3 & 250 & 22 & 287 \\
Week 4 & 600 & 48 & 679 \\
\hline \hline Time & \multicolumn{2}{c}{ Item 2 } \\
Periods & Mean & SD & RHS \\
\hline Week 1 & 500 & 23 & 538 \\
Week 2 & 400 & 46 & 476 \\
Week 3 & 600 & 50 & 683 \\
Week 4 & 850 & 61 & 951 \\
\hline \hline Time & \multicolumn{2}{c}{ Item 3 } \\
Periods & Mean & SD & RHS \\
\hline Week 1 & 450 & 28 & 497 \\
Week 2 & 450 & 20 & 483 \\
Week 3 & 350 & 29 & 398 \\
Week 4 & 600 & 35 & 658 \\
\hline \hline
\end{tabular}

In order to validate and verify the performance of the proposed CCP approach, a small test case is conducted involving the stochastic lot-sizing problem on parallel machines for production planning with four-week time periods $(T=4)$, three items $(i=3)$, and two machines $(j=2)$. The demands of each week of all items are assumed to be normally distributed and are shown mean, standard deviation (SD), and right hand side (RHS) of the constraints (9) in Table I. The demands must be integer number therefore the RHS values will be round up to the nearest value. The significance level is set at a constant $\left(\alpha_{i t}=0.05\right)$ for all items and periods. The initial quantities of inventory of all items are 420 units for item 1, 530 units for item 2, and 660 units for items 3. The remaining parameters of this numerical examples are set and shown in Table II.

TABLE II: PARAMETERS FOR NUMERICAL EXAMPLE

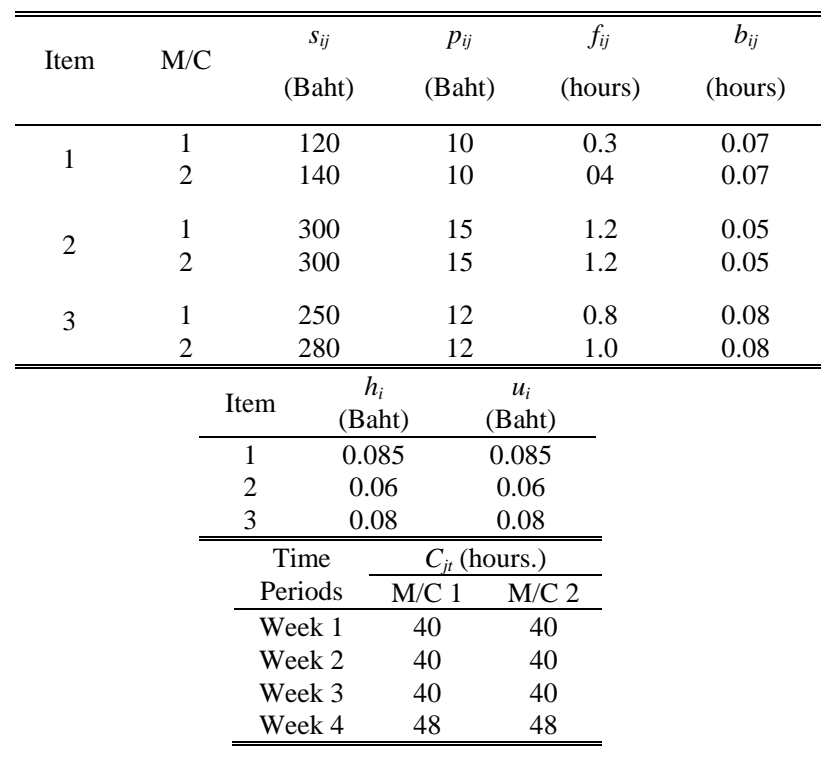

\section{B. Numerical Results}

The problem is run on a windows-based computer with an Intel@ Core(TM) i7-4500 U CPU @ 1.80GHz 2.40GHz CPU and 8GB Ram. The optimization software Gurobi Optimization for AMPL (Free Academic License) was used. The computing results found that the problem was solved in 0.0928 seconds and gave the feasible solution with total cost of $61,485.625$ Baht. The results of numerical example are shown in Table 3 . The results provide the producing quantity of each item on each machine in each period and this information can be used in the decision making process.

TABLE III: RESULTS OF NUMERICAL EXAMPLE

\begin{tabular}{ccccccc}
\hline \hline Time & \multicolumn{2}{c}{ Item 1 } & \multicolumn{2}{c}{ Item 2 } & \multicolumn{2}{c}{ Item 3 } \\
\cline { 2 - 6 } Periods & M/C1 & M/C2 & M/C1 & M/C2 & M/C1 & M/C2 \\
\hline Week 1 & 0 & 0 & 0 & 484 & 396 & 0 \\
Week 2 & 0 & 417 & 0 & 0 & 490 & 0 \\
Week 3 & 0 & 0 & 0 & 698 & 490 & 0 \\
Week 4 & 679 & 0 & 0 & 936 & 0 & 0 \\
\hline \hline
\end{tabular}

\section{CONCLUSIONS}

This research presented the CCP approach for the lot-sizing problem with stochastic demand on parallel machines. This method was used to transform uncertain constraints into deterministic constraints, which this equivalent deterministic formulation can be solved with basic software to find optimal solutions. The numerical example of the production planning of multiple items in a single stage capacitated lot-sizing problem in the setting of unrelated parallel machines with stochastic demand is conducted to show how to use the CCP approach to solve this problem. Computational results show its effectiveness. 


\section{REFERENCES}

[1] J. M. Bismut, "An introductory approach to duality in optimal stochastic control," Society for Industrial and Applied Mathematics Review, vol. 20, no. 1, pp. 62-78, Jan. 1978.

[2] X. Ding and C. Wang, "A novel algorithm of stochastic chance-constrained linear programming and its application," Mathematical Problems in Engineering, vol. 2012, pp. 1-17, July 2011.

[3] A. Charnes and W. W. Cooper, "Chance-constrained programming," Management Science, vol. 6, no. 1, pp. 73-79, Oct 1959.

[4] S. Ketsarapong, V. Punyangarm, K. Phusavat, and B. Lin, "An experience-based system supporting inventory planning: a fuzzy approach," Expert Systems with Applications, vol. 39, pp. 6994-7003, 2012.

[5] R. Kian, U. Gurler and E. Berk, "The dynamic lot-sizing problem with convex economic production costs and setups," International Journal Production Economics, vol. 155, pp. 361-379, Feb 2014.

[6] F. W. Harris, "How many parts to make at once," The Magazine of Management, vol. 10, no. 2, pp. 135-136, Feb 1913.

[7] H. M. Wagner and T. M. Whitin, "Dynamic version of the economic lot size model," Management Science, vol. 5, no. 1, pp. 89-96, Oct 1958.

[8] J. Mula, R. Poler, J. P. Garci'a-Sabater and F. C. Lario, "Models for production planning under uncertainty: A review," International Journal of Production Economics, vol. 103, no. 1, pp. 271-285, Sep 2006.

[9] E. A. Silver, "Inventory control under a probabilistic, time-varying, demand pattern," AIIE Transactions, vol. 10, no. 4, pp. 371-379, 1978.

[10] P. Kall and S. W. Wallace, Stochastic Programming, Wiley, Chichester, 1994.

[11] G. B. Dantzig, "Linear programming under uncertainty," Management Science, vol. 1, no. 3-4, pp. 197-206, April-July 1955.

[12] B. Liu. (May 2014). Introduction to uncertain programming. A rough manuscript for distribution in uncertainty theory laboratory only. [Online].

http://www.orsc.edu.cn/ zhoujian/research/course1/file/up.pdf

[13] J. H. Bookbinder and J. Y. Tan, "Strategies for the probabilistic lot-sizing problem with service-level constraints," Management Science, vol. 34, no. 9, pp. 1096-1108, Sep. 1988.

[14] S. A. Tarim and B. G. Kingsman, "The stochastic dynamic production/inventory lot-sizing problem with service-level constraints," International Journal Production Economics, vol. 88, pp. 105-119, Mar. 2004.

[15] R. Rossi, S. A. Tarim, B. Hnich and S. Prestwich, "A global chance-constraint for stochastic inventory systems under service level constraints," Constraints, vol. 13, no. 4, pp. 490-517, Feb 2008.
[16] A. A. Taleizadeh, S. T. A. Niakib, and S. M. H. Seyedjavadic, "Multi-product multi-chance-constraint stochastic inventory control problem with dynamic demand and partial back-ordering: A harmony search algorithm," Journal of Manufacturing Systems, vol. 31, no. 2, pp. 204-213, Apr 2012.

[17] S. Chotayakul, J. E. Kobza, J. Pichitlamken and P. Charnsethikul, “An optimization-based heuristic for capacitated lot-sizing inventory model with stochastic demand: case study of an ATM network," in Proc. 17th International Conference on Industrial Engineering: Theory, Applications and Practice, Pusan National University, Busan, Korea, 2013, pp. 695-703.

[18] J. R. Birge and F. Louveaux, Introduction to Stochastic Programming, Springer, New York, NY, 1997.

[19] J. L. Higle, J. C. Bean and R. L. Smith, "Deterministic equivalent in stochastic infinite horizon problems," Mathematics of Operations Research, vol. 15, no. 3, pp. 396-407, 1990.

[20] A. Ruszczynski, "Probabilistic programming with discrete distributions and precedence constrained knapsack polyhedral," Mathematical Programming, vol. 93, no. 2, pp. 195-215, Dec 2002.

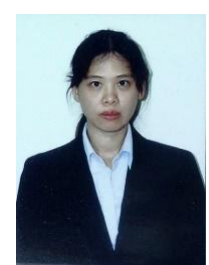

Supatchaya Chotayakul is an instructor in the Department of Industrial Engineering at Srinakharinwirot University, Ongkharak, Nakhonnayok, Thailand. She received her master degree and Ph.D. degree in industrial engineering at Kasetsart University. Her major field of study was dynamic lot-sizing problem. Her current research and teaching interests are in the inventory and warehouse management, engineering statistics, and operation

research.

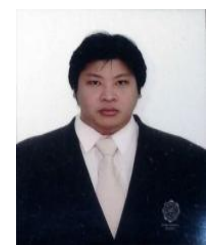

Varathorn Punyangarm is an instructor in the Department of Industrial Engineering at Srinakharinwirot University, Ongkharak, Nakhonnayok, Thailand. He received his master degree in master degree in the field of manufacturing system engineering at King Mongkut's University of Technology Thonburi, Bangkok, Thailand in 2001 and Ph.D, degree in industrial engineering at Kasetsart University, Bangkok, Thailand in 2008. His major field of study was data envelopment analysis (DEA) approach for performance evaluation in balances scorecard framework. He has published number of research papers in the field of lot-sizing problem model based fuzzy approach in inventory planning. His current teaching interests are in the engineering economics, quality management and logistics management. 